'Servicio de Cirugía Cardiaca. Instituto Nacional del Tórax. Santiago, Chile. ${ }^{2}$ Departamento de enfermedades Cardiovasculares. Clínica de la Universidad de los Andes. Santiago, Chile. ${ }^{3}$ Unidad de Cardiología Intervencional y Hemodinamia, Departamento de Cardiología, Hospital Sótero del Río. Santiago, Chile.

Trabajo no recibió financiamiento. Los autores declaran no tener conflictos de interés.

Recibido el 22 de febrero de 2021 , aceptado el 25 de mayo de 2021.

Correspondencia a: Dr. Gabriel Olivares R. Servicio de Cirugía Cardiaca. Instituto Nacional del Tórax. Santiago, Chile. golivarr@gmail.com

\section{Cirugía de revascularización miocárdica versus angioplastía en el tratamiento de la enfermedad coronaria de tres vasos y tronco de la coronaria izquierda}

\author{
GABRIEL OLIVARES R..$^{1,2}$, NICOLÁS VEAS ${ }^{2,3}$
}

\begin{abstract}
Recent randomized controlled trials confirmed the beneficial outcomes with coronary artery bypass grafting (CABG) compared with percutaneous coronary intervention (PCI) in patients with severe three-vessel coronary artery and left main disease. An increased long-term survival after $C A B G$ is associated with a reduction in spontaneous myocardial infarction and repeat revascularization rates. While PCI treats only flow-limiting lesions, CABG treats the whole coronary artery, preventing events in the future. Due to different clinical and anatomic factors affecting the outcomes, the heart team should formulate treatment assignment recommendations.
\end{abstract}

(Rev Med Chile 2021; 149: 1182-1188)

Key words: Cardiovascular Surgical Procedures; Coronary Artery Disease; Percutaneous Coronary Intervention.
$\mathrm{R}$ ecientemente, se han publicado los resultados alejados de importantes estudios aleatorizados controlados multicéntricos que comparan la cirugía de revascularización miocárdica (CABG) y angioplastia (PCI) en pacientes con enfermedad coronaria de tres vasos o del tronco coronario izquierdo (TCI). Estas publicaciones incluyen, el seguimiento a 10 años del estudio SYNTAX ${ }^{1}$, resultados de los estudios sobre enfermedad del TCI: EXCEL ${ }^{2}$ y $\mathrm{NOBLE}^{3}$ con 5 años de seguimiento y finalmente, el estudio de pacientes diabéticos con enfermedad coronaria FREEDOM con 7,5 años de seguimiento ${ }^{4}$.

Los resultados de estudios aleatorizados representan un enorme esfuerzo para definir cual es la mejor terapia para estos pacientes. Sus resultados modifican las recomendaciones de las guías clínicas y afectan la practica clínica, por lo que es crucial analizar cuidadosamente sus resultados.
La gran relevancia de estos hallazgos se enmarca en que en Chile, la primera causa de muerte son las enfermedades del sistema circulatorio con alrededor de 28.148 fallecidos por año, de éstos, 8.155 corresponden a enfermedades isquémicas del corazón ${ }^{5}$, sin mencionar aquellos que tienen limitada su vida cotidiana a consecuencia de la angina. La mortalidad anual en pacientes con enfermedad coronaria de tres vasos o TCI es de 6,6 a $8,2 \%{ }^{6}$, por lo que representa un importante problema de salud pública.

\section{Enfermedad coronaria de tres vasos}

El estudio SYNTAX fue un trabajo randomizado, controlado y multi-céntrico, que incluyó 1.800 pacientes, donde se compara la PCI con primera generación de stents fármaco-activos versus $\mathrm{CABG}$ 
en pacientes con enfermedad coronaria de tres $\operatorname{vasos}(\mathrm{n}=1.095)$ y TCI $(\mathrm{n}=705)$. Financiado por Boston Scientific hasta los 5 años de seguimiento, sin embargo, la extensión a 10 años significó un esfuerzo adicional de los investigadores (SYNTAXES) $)^{1}$. El objetivo de este trabajo fue realizar una evaluación de mortalidad general a 10 años de seguimiento. El resultado primario de mortalidad general ocurrió en $27 \%$ en el grupo PCI y $24 \%$ en el grupo CABG (HR 1,17; 95\% CI 0,97 a $1,41, p=0,092)$. Para el resultado secundario de mortalidad general con máximo seguimiento, la PCI fue asociada con mayor mortalidad (34\% PCI versus 29\% CABG; HR 1,18; 95\% CI 1,00 a 1,39).

El análisis de subgrupos previamente determinados demostró beneficios en supervivencia en el grupo de pacientes con enfermedad de tres vasos sometidos a CABG versus PCI. La mortalidad a 10 años en grupo PCI fue de $28 \%$ y $21 \%$ en el grupo CABG (HR 1,41; 95\% CI 1,10 a 1,80). En el subgrupo de enfermedad del TCI no hubo diferencia estadísticamente significativa.

Previamente publicado, la frecuencia de eventos adversos cardiacos mayores y cerebrovasculares (MACCE) a 5 años, fue de 37,3\% para PCI versus $26,9 \%$ para CABG $(\mathrm{p}<0,0001)$, determinado principalmente por un incremento de infartos miocárdicos (IM) y repetición de la revascularización durante el seguimiento, especialmente en pacientes con mayor complejidad anatómica ${ }^{7}$.

\section{Enfermedad del tronco de la coronaria izquierda}

El estudio EXCEL y NOBLE compararon PCI versus CABG en pacientes con enfermedad del TCI $\mathrm{y}$ otros vasos coronarios a 5 años de seguimiento.

El estudio EXCEL fue un trabajo aleatorizado controlado, multi-céntrico de no inferioridad, que incluyó 1.905 pacientes con enfermedad de TCI con baja o intermedia complejidad anatómica, financiado por Abbot. Destacaba, además, que pacientes con lesiones entre $50-70 \%$ de estenosis podían realizarse estudios funcionales hemodinámicos (estudio no invasivo con isquemia, FFR $\leq 0,80$ o IVUS $\leq 6 \mathrm{~mm}^{2}$ ) y con SYNTAX score menor a 32. El resultado primario compuesto (MACE) de muerte global, accidente cerebrovascular e IM no demostró diferencias significativas
(22\% PCI versus 19,2\% CABG; HR 1,19; 95\% CI 0,95 a 1,5). Sin embargo, los resultados secundarios demostraron importantes diferencias. El resultado secundario compuesto (MACCE) de muerte general, accidente cerebrovascular, IM y repetición de la revascularización fue de $31 \%$ para PCI versus $24,9 \%$ para CABG (HR 1,39; 1,13 a $1,7)$. Finalmente, lo más relevante del trabajo fue el análisis de mortalidad general que resultó mayor en el grupo PCI 13\% versus CABG 9,9\% (HR 1,38; $95 \%$ CI 1,03 a 1,85 ).

El análisis de la temporalidad de los resultados demostró como a partir del año de seguimiento CABG demuestra su superioridad para MACE, MACCE, muerte general, IM y repetición de revascularización, interesantemente, no existieron diferencias para accidente cerebrovascular.

\section{Controversia en la interpretación de los resultados}

Después de la publicación del estudio EXCEL, surgieron críticas a la interpretación de los resultados. Probablemente, acostumbrados a la naturaleza dicotómica en la interpretación de resultados, se concluyó que no existían diferencias entre CABG y PCI para el tratamiento de las lesiones del TCI. Dado la metodología del estudio, siendo este de no inferioridad, no se alcanzaron diferencias en el resultado primario. Para aclarar esta disputa y ayudarnos a la interpretación de los resultados, fue publicado recientemente un análisis bayesiano en JAMA internal medicine ${ }^{8}$. Los análisis bayesianos nos entregan estimaciones de probabilidades y han sido utilizados por muchos años en la interpretación de resultados. Este análisis sugiere con una alta probabilidad que PCI no sólo es estadísticamente inferior, sino también, clínicamente inferior a CABG tanto para el MACE, MACCE y muerte general. Así, el análisis estimó con $95 \%$ de probabilidad que la diferencia del MACE a 5 años estaba incrementada en el grupo PCI comparada con CABG y hubo $87 \%$ de probabilidades que esta diferencia fuera mayor a 1 evento extra por cada 100 pacientes tratados. Para mortalidad general, este análisis sugirió con $99 \%$ de probabilidad, que esta estaba aumentada en el grupo PCI comparada con CABG y hubo $94 \%$ de probabilidad de que esta diferencia absoluta excedió 1 muerte extra por cada 100 pacientes tratados. 
El estudio EXCEL, además, introdujo una definición bioquímica de IM. Los infartos peri-procedimiento fueron $6,1 \%$ en el grupo CABG comparado con 3,9\% en el grupo PCI (HR 0,63; 95\% CI, 0,41 a 0,96), sin embargo, esta diferencia en infarto peri-procedimiento, no se tradujo en diferencias de la mortalidad peri-procedimiento ( $1 \%$ PCI versus $1,1 \%$ CABG). En contraste, los IM durante el seguimiento fueron mayor en el grupo PCI 6,8\% versus $3,5 \%$ grupo CABG (HR 1,96; 95\% CI, 1,25 a 3,06), además de su probable asociación con la mortalidad general durante el seguimiento (entre 1 a 5 años post intervención) (10\% PCI versus $6,6 \%$ CABG, HR 1,57; 95\% CI 1,12 a 2,19). Motivo de controversia es que no se encontraron diferencias significativas en la mortalidad cardiovascular (95\% CI, OR 1,26 (0,85-1,85)), siendo la gran diferencia la mortalidad de causa indeterminada $(95 \%$ CI, OR 1,78 (0,78-4,06)).

El análisis de mortalidad general en un estudio de tratamiento TCI es sumamente relevante, ya que la oclusión del stent o puente en ese lugar anatómico puede ser devastador, puede ocurrir en forma súbita o darse en el contexto de estados mórbidos concomitantes. Previamente, ha sido discutido las dificultades en la asignación de mortalidad, sesgo y/o error en la interpretación de la causa de mortalidad, sumado al hecho de una baja realización de autopsias en estudios de patología cardiovascular'. Así, este efecto sobre la mortalidad general no puede ser obviado del análisis final del trabajo, ya que representa el resultado clínico mas relevante en un trabajo de esta naturaleza. Claro está que este es un resultado experimental, dado que el estudio no fue diseñado para demostrar no inferioridad en términos de mortalidad.

El estudio NOBLE fue otro trabajo aleatorizado controlado, multi-céntrico, financiado por Aarhus University Hospitals y Biosensors. Este incluyó 1.201 pacientes con enfermedad de TCI > 50\% o con estudios de funcionalidad hemodinámica alterados (FFR $\leq 0,80)$. Con un promedio de seguimiento de 4,9 años, el MACCE fue de $28 \%$ para PCI y $19 \%$ para CABG (HR 1,58 95\%; CI 1,24 a $2,01 ; p=0,0002)$. Esta diferencia determinada por la mayor frecuencia de IM durante el seguimiento en el grupo PCI ( $8 \%$ PCI versus 3\% CABG, HR 2,$99 ; 95 \%$ CI 1,66 a 5,$39 ; \mathrm{p}=0,0002$ ) y una mayor frecuencia en la repetición de revascularización en el grupo PCI (17\% grupo PCI versus $10 \%$ grupo CABG, HR 1,73; 95\% CI 1,25 a 2,40; $\mathrm{p}=0,0009)$.
No existieron diferencias en mortalidad general $\mathrm{y}$ accidentes cerebrovasculares. Es posible que las diferencias en el resultado primario, en comparación con Excel, se deba a distintas definiciones.

\section{Enfermedad multivaso y diabetes}

En el grupo de pacientes diabéticos, recientemente fueron publicados los resultados del trabajo FREEDOM, estudio aleatorizado controlado multi-céntrico, financiado por National Heart, Lung, and Blood Institute (NHLBI). Incluyó 1.900 pacientes diabéticos con enfermedad coronaria de 2 o más vasos con estenosis de mas de $70 \%$. Los resultados a 5 años habían sido publicados anteriormente y demostraron inferioridad para el MACE en el grupo PCI versus CABG $(26,6 \% \mathrm{PCI}$ versus $18,7 \%$ CABG, $p=0,005)$, estas diferencias fueron debidas a una mayor frecuencia de IM en el grupo $\mathrm{PCI}^{10}$. Sin embargo, ahora con promedio de seguimiento de 7,5 años y el $49,6 \%$ de la cohorte original de pacientes, la mortalidad general fue significativamente mayor en el grupo PCI versus CABG (24,3\% PCI versus 18,3\% CABG, HR 1,36; $95 \%$ CI 1,07 a 1,$74 ; \mathrm{p}=0,01)$.

\section{Explicación de las diferencias entre PCI versus CABG}

La enfermedad coronaria producida por la formación de placas de ateroesclerosis provoca grados variables de estenosis, lo que puede producir angina o un IM, debido a la ruptura de la placa y oclusión trombótica del vaso ${ }^{11}$.

Diferentes trabajos han demostrado a largo plazo, la superioridad de CABG en reducir la frecuencia de infartos miocárdicos y la necesidad de repetir revascularización ${ }^{2,3,7,12}$, excepto en las lesiones menos complejas (SYNTAX $<22)^{7}$. Durante el seguimiento, pacientes con lesiones de TCI tienen una frecuencia de IM entre 6 a $8 \%$ para grupo PCI versus 3 a 3,5\% para el grupo $\mathrm{CABG}^{2,3}$. Para pacientes con enfermedad coronaria de tres vasos la frecuencia de IM fue de 10,6 para el grupo $\mathrm{PCI}$ versus 3,6 para el grupo $\mathrm{CABG}^{12}$. En pacientes diabéticos estos riesgos se duplican (IM 13,9\% grupo PCI versus $6 \%$ grupo CABG $)^{10}$.

Esta prevención en IM durante el seguimiento puede explicar las diferencias en mortalidad 
observadas durante seguimientos más extensos, atribuyendo así el efecto protector de la $\mathrm{CABG}^{13}$. Por otra parte, estudios de PCI en síndromes coronarios agudos han demostrado que los IM durante el seguimiento son un poderoso predictor independiente de mortalidad y deberían considerarse en forma distinta al infarto peri-procedimiento ${ }^{14}$, apoyando los resultados del estudio EXCEL, con respecto al distinto factor pronóstico de infartos peri-procedimiento versus infartos espontáneos durante el seguimiento ${ }^{2}$.

La repetición de revascularización también fue menor con CABG versus PCI. El estudio SYNTAX y EXCEL demostraron una mayor frecuencia en las reintervenciones con $\mathrm{PCI}^{2,7}$. La necesidad repetición de revascularización se debió en su mayoría a progresión de la enfermedad ateroesclerótica del vaso afectado y de menor manera a otros vasos ${ }^{15,16}$. En SYNTAX la repetición de revascularización en el grupo PCI ocurrió entre el 23 y el 30,9\% de los pacientes, dependiendo de la complejidad anatómica versus $12 \%$ en el grupo CABG. En EXCEL la frecuencia de reintervenciones fue de 17,2 en el grupo PCI versus 10,5 en el grupo CABG, similares cifras fueron reportadas en el estudio NOBLE.

Esta mayor frecuencia de reintervención fue un predictor independiente de infarto, accidente cerebrovascular y muerte en SYNTAX ${ }^{15}$, además se asoció a un incremento en la mortalidad en EXCEL $^{16}$, por lo que las reintervenciones no deben ser vistas como procedimientos complementarios sin riesgos. Esta menor frecuencia de repetición de la revascularización de CABG comparada con PCI, podría ser otro de los mecanismos que expliquen la menor mortalidad a largo plazo ${ }^{15,16}$. Otra posible explicación, es que la progresión de enfermedad proximal al bypass es más rápida y más severa en la arteria nativa. Esto ha llevado a la hipótesis de que pacientes con revascularización quirúrgica, son malos candidatos a una intervención de revascularización percutánea a posteriori, sin embargo faltan estudios que confirmen esta hipótesis.

Mientras PCI trata lesiones específicas de una arteria coronaria, CABG trata la arteria coronaria completa. Estudios de imágenes han demostrado que la mayoría de los infartos ocurren en la porción proximal del vaso coronario y que la ubicación de los puentes en CABG ocurren distal a la porción mas frecuentemente afectada por los síndromes coronarios agudos, demostrado la naturaleza preventiva del puente ${ }^{17}$.
Otro mecanismo importante para considerar al momento de elegir estrategia, que explica las diferencias en mortalidad global entre PCI y CABG, es la capacidad de conseguir una revascularización completa. La PCI se asocia con menores tasa de revascularización completa, probablemente afectada por factores anatómicos. En el estudio SYNTAX, la revascularización completa fue de 56,7\% en el grupo PCI versus 63,2\% en el grupo CABG $(\mathrm{p}=0,005)^{18}$. El efecto de una revascularización incompleta se aprecia en el pronóstico a largo plazo, mientras mayor sea el SYNTAX residual menor será la supervivencia ${ }^{19}$.

Recientemente publicado, un metaanálisis con 4.490 pacientes de los 5 mayores ensayos clínicos sobre PCI versus CABG en revascularización de TCI, no se encontraron diferencias significativas para mortalidad global (95\% CI OR 1,09; 0,88$1,34, \mathrm{p}: 0,42)$ ni para mortalidad cardiovascular (95\% CI OR 1,14; 0,88-1,47, p: 0,31). Las diferencias si fueron significativas en términos de necesidad de nueva revascularización (95\% CI OR 1,82; $1,49-2,22, \mathrm{p}<0,0001)$, pero no en infarto agudo al miocardio (95\% CI OR 1,52; 0,98-2,37, p: 0,06) ni en accidente cerebrovascular (95\% CI OR 0,84; $0,48-1,45)^{17}$. Con respecto a la necesidad de nueva revascularización como un resultado en los diversos ensayos clínicos, este es un resultado que puede ser debatible dado que es un médico quien envía al paciente a nuevo estudio y no es un resultado clínico espontáneo, además es incierto si repercute en la mortalidad o en infartos posteriores ${ }^{27,28}$.

Finalmente, además de factores clínicos antes mencionados, CABG logra un mejor alivio de la angina, en pacientes con enfermedad coronaria de tres vasos. A 5 años $22 \%$ de los pacientes sometidos a PCI tiene angina versus $9,6 \%$ en el grupo CABG $(\mathrm{p}<0,001)^{12}$.

\section{Ajustando la evidencia a la práctica clínica}

El análisis de la temporalidad de los resultados de los trabajos previamente analizados se desprende que a partir del año de seguimiento se comienzan a ver los efectos positivos de CABG, disminuyendo los infartos y la necesidad de repetición de reintervenciones. Durante el seguimiento alejado comienzan a separarse las curvas de mortalidad favorables a $\mathrm{CABG}^{2,3,4,7}$. Resulta interesante que en el estudio NOBLE no se observa una separación de las curvas a 5 años de seguimiento (HR 1,08 p: 0,68). 
En el registro SYNTAX de aquellos pacientes enrolados, pero no aleatorizados, el motivo principal por la que un paciente fue asignado a CABG fueron las complejidades anatómicas (92\% en registro SYNTAX); mientras que el principal motivo por la que un paciente fue derivado a PCI fueron múltiples comorbilidades $(70 \%$ registro SYNTAX) ${ }^{18}$.

Los riesgos promedios de los trabajos deben ser contextualizados a los riesgos individuales de cada paciente. Así, podemos decir que CABG es afectado por las comorbilidades del paciente y expectativas de vida, por otro lado, PCI es afectado por las complejidades anatómicas de los vasos coronarios. Pacientes con buenas expectativas de vida, con lesiones coronarias complejas o diabéticos se beneficiarán de CABG; pacientes con limitada expectativa de vida o con múltiples comorbilidades y situaciones de emergencia, se beneficiarán de PCI.

Un aspecto quirúrgico relevante y que debemos destacar es que aquellos pacientes que obtendrán mayor beneficio de la revascularización por esta técnica son aquellos a los que se les ofrecerá el uso de injertos arteriales, especialmente el uso de arteria mamaria interna a la descendente anterior. Probablemente uno de los factores que incideron en el resultado de los estudios mencionados, es el alto uso de arterias torácicas internas bilaterales para la revascularización (27\% en SYNTAX y 28\% en EXCEL). Existen numerosos estudios observaciones que demuestran una mejor supervivencia con el uso de un segundo injerto arterial, sin embargo, estos beneficios se hacen evidentes mas allá de los 10 años de seguimiento ${ }^{25,26}$.

Por otro lado, es muy importante analizar estos datos estadísticos con las realidades locales. El resultado de CABG no solo depende del cirujano, sino además de las distintas unidades de apoyo, tales como: anestesista cardiovascular, unidades de paciente intensivo, terapia transfusional, etc. A diferencia de cirugía, los resultados de la PCI dependen más del operador $y$, en general, no requiere de grandes cuidados a posterior. Intuitivamente es fácil pensar que aquellos centros y operadores con mayor volumen tienen mejores resultados a corto y largo plazo. Los resultados de PCI son reproducibles, dado que la técnica está cada día más protocolizada y estandarizada. La tecnología ha ido avanzando, sobretodo los últimos 5 años. Las distintas generaciones de stents, han permitido disminuir las tasas de trombosis y de reestenosis, así como las imágenes intracoronarias han logrado optimizar las técnicas de implantes, además el uso de fisiología coronaria ha permitido evitar el tratamiento, con su riesgo asociado, de placas que no lo requieren desde el punto de vita funcional. Es por ello, que los análisis y ensayos clínicos que sigan apareciendo, probablemente cambien los resultados en términos de revascularización post evento índice.

La evidencia ha demostrado que pacientes con enfermedad coronaria de tres vasos, en que, pese a que de acuerdo a las guías clínicas y recomendaciones se beneficiarían de CABG, finalmente reciben una $\mathrm{PCI}^{20,21}$. Probablemente uno de los

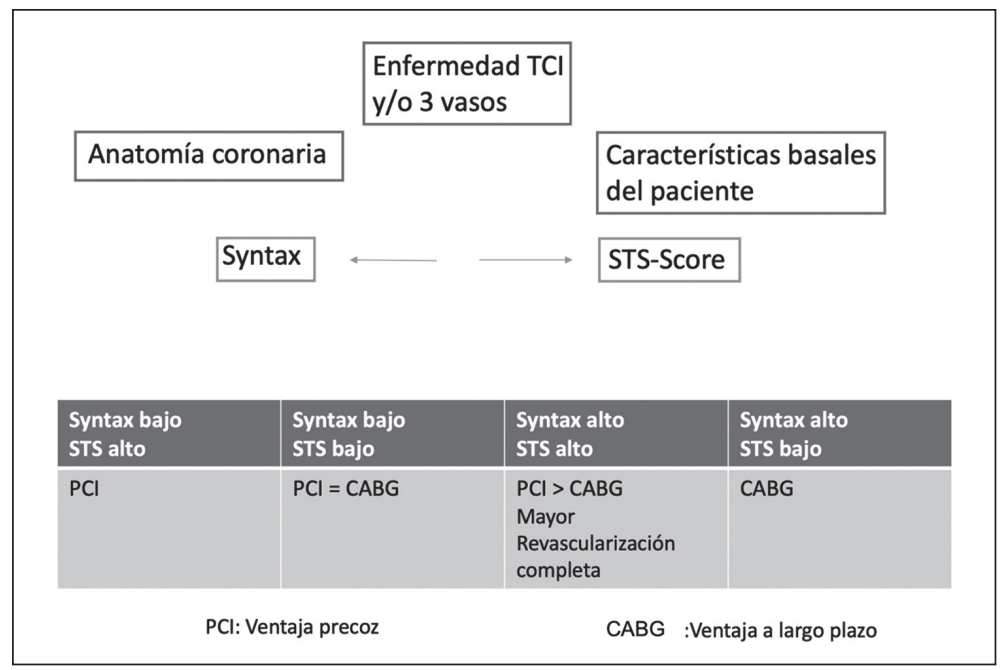

Figura 1. Algoritmo simple para identificar estrategia de revascularización entre ambas técnicas, con énfasis en el Syntax score y el STS-score. PCl: angioplastía coronaria. CABG: cirugía de revascularización miocárdica. 
conceptos más importantes de estos trabajos fue la introducción del concepto de decisión en equipo (Heart Team). Este equipo incluye un cardiólogo clínico, cardiólogo intervencionista y al cirujano cardiaco. Esta estrategia se considera la mejor forma de evaluar las ventajas y desventajas de cada tratamiento, incluso podría reasignar estrategias en $30 \%$, siendo la más frecuente al manejo médi$\mathrm{CO}^{22}$. Con esta aproximación se logra: (a) una mejor interpretación de los exámenes diagnósticos, (b) implementar un tratamiento acorde a las guías clínicas, (c) considerar la realidad y resultados de cada institución, e (d) incluye las preferencias del paciente ${ }^{22}$. Pero no es responsabilidad exclusiva del equipo de cardiología que realiza el estudio y diagnostico, deberá ser responsabilidad del equipo de cirujanos estar disponibles para la discusión, además de facilitar y gestionar una derivación y resolución expedita a CABG.

Por último, otra de las lecciones de estos trabajos, fue la alta tasa de adherencia al tratamiento médico, tanto para el grupo PCI como para $\mathrm{CABG}^{2,18}$. La prevención secundaria cardiovascular es uno de los componentes más importantes para el pronóstico a largo plazo, recordando que muchos infartos se producirán de placas de ateroesclerosis no hemodinámicamente significativas $^{14,23,24}$.

\section{Conclusión}

En comparación con PCI, evidencia reciente concluye que CABG disminuye la ocurrencia de IM y repetición de revascularización durante el seguimiento, además, de disminuir la mortalidad en aquel paciente con enfermedad de tres vasos y TCI. Transferir los resultados de los estudios a riesgos individuales para ajustar el tratamiento a cada paciente en particular, es sin duda responsabilidad en todos los que participamos en el diagnostico y tratamiento de estos pacientes, siendo necesario un enfoque multidisciplinario para optimizar los resultados a largo plazo.

\section{Referencias}

1. Thuijs DJFM, Kappetein AP, Serruys PW, Mohr FW, Morice MC, Mack MJ, et al. Percutaneous coronary intervention versus coronary artery bypass grafting in patients with three-vessel or left main coronary artery disease: 10-year follow-up of the multicentre randomised controlled SYNTAX trial. Lancet 2019; 394(10206): 1325-34.

2. Stone GW, Kappetein AP, Sabik JF, Pocock SJ, Morice MC, Puskas J, et al. Five-Year Outcomes After PCI or CABG for Left Main Coronary Disease. N Engl J Med 2019; 381(19): 1820-30.

3. Holm NR, Mäkikallio T, Lindsay MM, Spence MS, Erglis A, Menown IBA, et al. Percutaneous Coronary Angioplasty Versus Coronary Artery Bypass Grafting in the Treatment of Unprotected Left Main Stenosis: Updated 5-year Outcomes From the Randomised, Non-Inferiority NOBLE Trial. Lancet 2020; 395(10219): 191-9.

4. Farkouh ME, Domanski M, Dangas GD, Godoy LC, Mack MJ, Siami FS, et al. Long-Term Survival Following Multivessel Revascularization in Patients With Diabetes: The FREEDOM Follow-On Study. J am Coll Cardiol 2019; 73(6): 629-38.

5. INE, Anuario estadísticas vitales, informe anual, 2016.

6. Fihn SD, Blankenship JC, Alexander KP, Bittl JA, Byrne JG, Fletcher BJ, et al. 2014 ACC/AHA/AATS/ PCNA/SCAI/STS focused update of the guideline for the diagnosis and management of patients with stable ischemic heart disease: a report of the American College of Cardiology/American Heart Association Task Force on Practice Guidelines, and the American Association for Thoracic Surgery, Preventive Cardiovascular Nurses Association, Society for Cardiovascular Angiography and Interventions, and Society of Thoracic Surgeons. J Am Coll Cardiol 2014; 64(18): 1929-49.

7. Mohr FW, Morice MC, Kappetein AP, Feldman TE, Ståhle E, Colombo A, et al. Coronary artery bypass graft surgery versus percutaneous coronary intervention in patients with three-vessel disease and left main coronary disease: 5-year follow-up of the randomised, clinical SYNTAX trial. Lancet 2013; 381: 629-38.

8. Brophy JM. Bayesian Interpretation of the EXCEL Trial and Other Randomized Clinical Trials of Left Main Coronary Artery Revascularization. JAMA Intern Med 2020; 180(7): 1-7.

9. Lauer MS, Blackstone EH, Young JB, Topol EJ. Cause of death in clinical research: time for a reassessment? J Am Coll Cardiol 1999; 34(3): 618-20.

10. Farkouh ME, Domanski M, Sleeper LA, Siami FS, Dangas G, Mack M, et al. Strategies for Multivessel Revascularization in Patients with Diabetes. N Engl J Med 2012; 367: 2375-84.

11. Thygesen K, Alpert JS, Jaffe AS, Chaitman BR, Bax JJ, Morrow DA, et al. Executive Group on behalf of the 
Joint European Society of Cardiology (ESC)/American College of Cardiology (ACC)/American Heart Association (AHA)/World Heart Federation (WHF) Task Force for the Universal Definition of Myocardial Infarction. Fourth Universal Definition of Myocardial Infarction (2018). J Am Coll Cardiol 2018; 72(18): 2231-64.

12. Head SJ, Davierwala PM, Serruys PW, Redwood SR, Colombo A, Mack MJ, et al. Coronary artery bypass grafting vs. percutaneous coronary intervention for patients with three-vessel disease: final five-year follow-up of the SYNTAX trial. Eur Heart J 2014; 35(40): 2821-30.

13. Doenst T, Haverich A, Serruys P, Bonow RO, Kappetein P, Falk V, et al. PCI and CABG for Treating Stable Coronary Artery Disease: JACC Review Topic of the Week. J Am Coll Cardiol 2019; 73(8): 964-76.

14. Prasad A, Gersh BJ, Bertrand ME, Lincoff AM, Moses JW, Ohman EM, et al. Prognostic Significance of Periprocedural Versus Spontaneously Occurring Myocardial Infarction After Percutaneous Coronary Intervention in Patients With Acute Coronary Syndromes An Analysis From the ACUITY (Acute Catheterization and Urgent Intervention Triage Strategy) Trial. J Am Coll Cardiol 2009; 54(5): 477-86.

15. Parasca CA, Head SJ, Milojevic M, Mack MJ, Serruys PW, Morice MC, et al. Incidence, Characteristics, Predictors, and Outcomes of Repeat Revascularization After Percutaneous Coronary Intervention and Coronary Artery Bypass Grafting The SYNTAX Trial at 5 Years. J Am Coll Cardiol Intv 2016; 9: 2493-507.

16. Giustino G, Mehran R, Serruys PW, Sabik JF 3rd, Milojevic M, Simonton CA, et al. Mortality After Repeat Revascularization Following PCI or Coronary Artery Bypass Grafting for Left Main Disease: The EXCEL Trial. JACC Cardiovasc Interv 2020; 13(3): 375-87.

17. Jeon C, Candia SC, Wang JC, Holper EM, Ammerer M, Kuntz RE, et al. Relative spatial distributions of coronary artery bypass graft insertion and acute thrombosis: A model for protection from acute myocardial infarction. Am Heart J 2010; 160: 195-201.

18. Serruys PW, Morice MC, Kappetein AP, Colombo A, Holmes DR, Mack MJ, et al. Percutaneous Coronary Intervention versus Coronary-Artery Bypass Grafting for Severe Coronary Artery Disease. N Engl J Med 2009; 360: 961-72.

19. Farooq V, Serruys PW, Bourantas CV, Zhang Y, Muramatsu T, Feldman T, et al. Quantification of Incomplete Revascularization and its Association With Five-Year Mortality in the Synergy Between Percutaneous Coronary Intervention With Taxus and Cardiac Surgery (SYNTAX) Trial Validation of the Residual SYNTAX Score. Circulation 2013; 128: 141-51.
20. Chan PS, Rao SV, Bhatt DL, Rumsfeld JS, Gurm HS, Nallamothu BK, et al. Patient and Hospital Characteristics Associated With Inappropriate Percutaneous Coronary Interventions. J Am Coll Cardiol 2013; 62: 2274-81.

21. Stanetic BM, Ostojic M, Campos CM, Marinkovic J, Farooq V, Kovacevic-Preradovic T, et al. ApPropriateness of myocaRdial Revascularization assessed by the SYNTAX score II in a coUntry without cardiac Surgery faciliTies; PROUST study. Int J Cardiol 2017; 227: 47884.

22. Head SJ, Kaul S, Mack MJ, Serruys PW, Taggart DP, Holmes DR Jr, et al. The rationale for Heart Team decision-making for patients with stable, complex coronary artery disease. Eur Heart J 2013; 34(32): 2510-8.

23. Giroud D, Li JM, Urban P, Meier B, Rutishauer W. Relation of the site of acute myocardial infarction to the most severe coronary arterial stenosis at prior angiography. Am J Cardiol 1992; 69: 729-32.

24. Ambrose JA, Tannenbaum MA, Alexopoulos D, Hjemdahl-Monsen CE, Leavy J, Weiss M, et al. Angiographic progression of coronary artery disease and the development of myocardial infarction. J Am Coll Cardiol 1988; 12: 56-62.

25. Lytle BW, Blackstone EH, Loop FD, Houghtaling PL, Arnold JH, Akhrass R, et al. Two internal thoracic artery grafts are better than one. J Thorac Cardiovasc Surg 1999; 117: 855-72.

26. Tatoulis J, Buxton BF, Fuller JA. The right internal thoracic artery: the forgotten conduit - 5,766 patients and 991 angiograms. Ann Thorac Surg 2011; 92: 9-15.

27. Garg A, Rout A, Raheja H, Hakeem H, Sharma S. Long-term follow-up of percutaneous coronary intervention versus coronary artery bypass grafting in left main coronary artery disease: A systematic review and meta-analysis. Catheter Cardiovasc Inter 2020; Oct 26. Epub ahead of print. PMID: 33103847.

28. Lamelas P, Belardi G, Whitlock R, Stone G. Limitations of repeat revascularization as an outcome measure. JACC Review Topic of the Week. J Am Coll Cardiol. 2019; 74 (25): 3164-73.

29. Rashid M, Sperrin M, Ludman PF, O'Neill D, Nicholas $\mathrm{O}$, de Belder MA, Mamas MA. Impact of operator volume for percutaneous coronary intervention on clinical outcomes: what do the numbers say? Eur Heart J Qual Care Clin Outcomes. 2016; 2 (1): 16-22.

30. Tsang MB, Schwalm JD, Gandhi S, Sibbald MG, Gafni A, Mercuri M, et al. Comparison of Heart Team vs Interventional Cardiologist Recommendations for the Treatment of Patients With Multivessel Coronary Artery Disease. JAMA Netw Open. 2020; 3 (8): e2012749. 